\title{
A Two-Time-Scale Framework to Supervisory Predictive Control of an Integrated Wind/Solar Energy Generation and Water Desalination System
}

\author{
Wei Qi, Jinfeng Liu and Panagiotis D. Christofides
}

\begin{abstract}
This work proposes a two-time-scale framework for the design of a supervisory predictive control system for long-term optimal management and operation of an integrated wind-solar energy generation and reverse-osmosis (RO) water desalination system. Specifically, we design the supervisory control system via model predictive control (MPC) which coordinates the wind and solar subsystems as well as a battery bank to provide enough energy to the RO subsystem. In the supervisory MPC design, a two-time-scale property of the dynamics of the integrated system is taken advantage of to improve the computational efficiency of the control problem formulation. Furthermore, optimality considerations on system operation and energy savings are taken into account via appropriate constraints in the supervisory controller formulation. Simulations are carried out to illustrate the applicability and effectiveness of the proposed supervisory predictive control design.
\end{abstract}

\section{INTRODUCTION}

Alternative energy technologies, like wind/solar-based energy generation systems, are receiving national and worldwide attention owing to the rising rate of consumption of fossil fuels. In particular, drivers for solar/wind renewable energy generation systems are the environmental benefits, reduced investment risk, fuel diversification and energy autonomy, increased energy efficiency as well as potential increase of power quality and reliability, and in certain cases, potential grid expansion deferral due to the possibility of generation close to demand. On the other hand, reverse osmosis (RO) membrane desalination has emerged as one of the leading methods for water desalination due to the low cost and energy efficiency of the process (e.g., [1]).

Renewable energy sources, for example wind and solar energy, are attractive choices for providing energy to RO desalination systems for small communities in remote areas and isolated islands that have access to sea- or brackishwater. In the past few years, several studies have been done on the integration of renewable energy generation systems with RO desalination systems including wind-powered and solar-powered desalination systems (e.g., [2]). However, the combination of renewable energy sources and water desalination systems requires addressing challenges in the operation of the integrated system.

Wei Qi, Jinfeng Liu and Panagiotis D. Christofides are with the Department of Chemical and Biomolecular Engineering, University of California, Los Angeles, CA 90095-1592, USA. Panagiotis D. Christofides is also with the Department of Electrical Engineering, University of California, Los Angeles, CA 90095-1592, USA. Email: qiwei.0216@gmail.com, jinfeng@ucla.edu,pdc@seas.ucla.edu.
With respect to previous results on control of wind and solar systems, most of the efforts have focused on control of stand-alone wind (e.g., [3]) or solar systems (e.g., [4]). With respect to the control of $\mathrm{RO}$ water desalination systems, a nonlinear model-based control technique was recently proposed to deal with large set-point changes and variations in feed water salinity and was experimentally implemented [5]. In [6], we proposed a supervisory predictive control method for stand-alone wind-solar energy generation systems in which the supervisory control system was designed via model predictive control (MPC) to take into account optimal allocation of generation assignment between the two subsystems. In [7], we designed a supervisory predictive control system for a stand-alone wind-solar energy generation system which provides energy to a RO water desalination system. However, the results obtained in [6], [7] focused on short-term system operation and the approach adopted there cannot be extended to long-term operation because of the high computational burden involved in solving the full, integrated system nonlinear dynamic model.

In this work, we focus on the design of a supervisory control system for long-term optimal management and operation of an integrated wind-solar energy generation and $\mathrm{RO}$ water desalination system. The supervisory MPC coordinates the wind and solar subsystems as well as a battery bank to provide enough energy to the RO subsystem so that enough permeate water can be produced to satisfy the overall water consumption and storage demands. In the supervisory MPC design, a two-time-scale property of the dynamics of the integrated system is taken advantage of to improve the computational efficiency of the control problem formulation. Furthermore, optimality considerations on system operation and energy savings are taken into account via appropriate constraints in the supervisory controller formulation. Simulations are carried out to illustrate the applicability and effectiveness of the proposed method.

\section{INTEGRATED SYSTEM DESCRIPTION}

In this section, we present the modeling of the integrated energy generation and RO water desalination system. A schematic of the integrated system is shown in Fig. 1. In this system, the wind-solar energy generation system and the battery bank provide energy to the RO water desalination system. In this work, we assume that the integrated system is not connected to the electrical grid. 


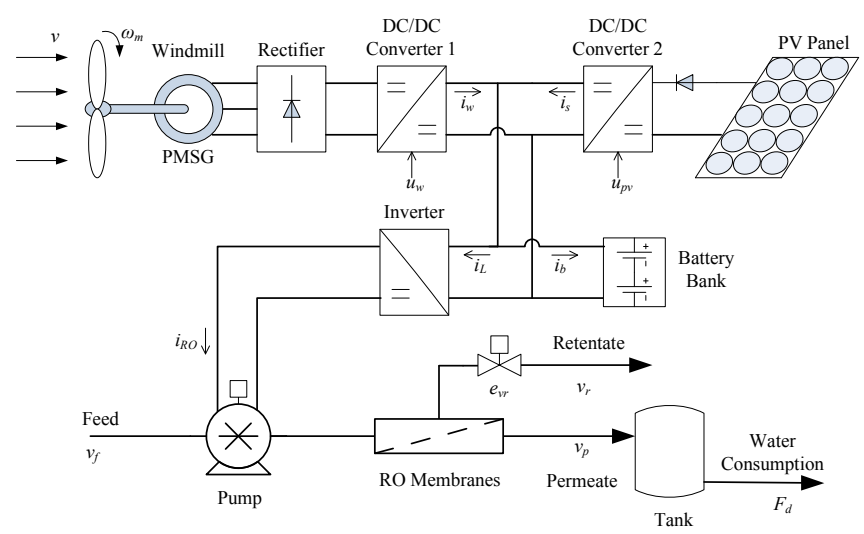

Fig. 1. Integrated wind/solar energy generation and water desalination system.

\section{A. Energy generation system description}

In the energy generation system, there are three subsystems: a wind generation subsystem, a solar generation subsystem and a lead-acid battery bank which is used to overcome periods of scarce generation.

In the wind generation subsystem, there is a windmill, a multipolar permanent-magnet synchronous generator (PMSG), a rectifier, and a DC/DC converter to interface the generator with the DC bus. The converter is used to control indirectly the operating point of the wind turbine (and consequently its power generation) by commanding the voltage on the PMSG terminals.

The mathematic description of the wind subsystem written in a rotor reference frame is as follows [9]:

$$
\begin{aligned}
\dot{i}_{q} & =-\frac{r_{s}}{L} i_{q}-\omega_{e} i_{d}+\frac{\omega_{e} \phi_{s r}}{L}-\frac{\pi v_{b} i_{q} u_{w}}{3 \sqrt{3} L \sqrt{i_{q}^{2}+i_{d}^{2}}} \\
\dot{i}_{d} & =-\frac{r_{s}}{L} i_{d}-\omega_{e} i_{q}-\frac{\pi v_{b} i_{d} u_{w}}{3 \sqrt{3} L \sqrt{i_{q}^{2}+i_{d}^{2}}} \\
\dot{\omega}_{e} & =\frac{P}{2 J}\left(T_{t}-\frac{3}{2} \frac{P}{2} \phi_{s r} i_{q}\right)
\end{aligned}
$$

where $i_{q}$ and $i_{d}$ are the quadrature current and the direct current in the rotor reference frame, respectively, $r_{s}$ and $L$ are the per phase resistance and inductance of the stator windings, respectively, $\omega_{e}$ is the electrical angular speed, $\phi_{s r}$ is the flux linked by the stator windings; $v_{b}$ is the voltage on the battery bank terminals, $u_{w}$ is the control signal (duty cycle of the DC/DC converter (DC/DC Converter 1 in Fig. 1)), $P$ is the PMSG number of poles, $J$ is the inertial of the rotating parts and $T_{t}$ is the wind turbine torque. The wind turbine torque can be written as:

$$
T_{t}=\frac{1}{2} C_{t}(\lambda) \rho A R v^{2}
$$

where $\rho$ is the air density, $A$ is the turbine-swept area, $R$ is the turbine radius, $v$ is the wind speed, and $C_{t}(\lambda)$ is a nonlinear torque coefficient which depends on the tip speed ratio $\left(\lambda=\frac{R \omega_{m}}{v}\right.$ with $\omega_{m}=\frac{2 \omega_{e}}{P}$ being the angular shaft speed).

Based on Eq. 1, we can express the power generated by the wind subsystem and injected into the DC bus as follows:

$$
P_{w}=i_{w} v_{b}
$$

where $i_{w}=\frac{\pi}{2 \sqrt{3}} \sqrt{i_{q}^{2}+i_{d}^{2}} u_{w}$ denotes the current which is injected to the DC bus by the wind subsystem.

In the solar subsystem, there is a photo-voltaic (PV) panel array and a half-bridge buck DC/DC converter (DC/DC Converter 2 in Fig. 1). The solar subsystem is connected to the DC bus via the DC/DC converter. In this subsystem, similar to the wind subsystem, the converter is used to control the operating point of the PV panels. The mathematic description of the solar subsystem is as follows [10]:

$$
\begin{aligned}
\dot{v}_{p v} & =\frac{i_{p v}}{C}-\frac{i_{s}}{C} u_{p v} \\
\dot{i}_{s} & =-\frac{v_{b}}{L_{c}}+\frac{v_{p v}}{L_{c}} u_{p v} \\
i_{p v} & =n_{p} I_{p h}-n_{p} I_{r s}\left(\exp \left(\frac{q\left(v_{p v}+i_{p v} R_{s}\right)}{n_{s} A_{c} K T}\right)-1\right)
\end{aligned}
$$

where $v_{p v}$ is the voltage level on the PV panel array terminals, $i_{s}$ is the current injected on the DC bus, $C$ and $L_{c}$ are electrical parameters of the buck converter, $u_{p v}$ is the control signal (duty cycle), $i_{p v}$ is the current generated by the $\mathrm{PV}$ array, $R_{s}$ is the series resistance in the PV circuit, $n_{s}$ is the number of PV cells connected in series, $n_{p}$ is the number of series strings in parallel, $K$ is the Boltzman constant, $A_{c}$ is the cell deviation from the ideal $p-n$ junction characteristic, $I_{p h}$ is the photocurrent, and $I_{r s}$ is the reverse saturation current. The power injected by the PV solar module into the DC bus can be computed by:

$$
P_{s}=i_{s} v_{b}
$$

Note that this power indirectly depends on the control signal $u_{p v}$.

The lead-acid battery bank may be modeled as a voltage source $E_{b}$ connected in series with a resistance $R_{b}$ and a capacitance $C_{b}$. Based on this simple model, the DC bus voltage expression can be written as follows:

$$
v_{b}=E_{b}+v_{c}+i_{b} R_{b}
$$

where $i_{b}$ is the current across the battery bank, $v_{c}$ is the voltage in capacitor $C_{b}$ and its dynamics is as follows:

$$
\dot{v}_{c}=\frac{1}{C_{b}} i_{b}
$$

The quantity of electricity stored in the battery bank can be calculated as follows:

$$
Q_{c}=C_{b} v_{c}
$$

Based on Eq. 8, the state of charge (SOC) of the battery bank, $s_{b}$, can be calculated as follows:

$$
s_{b}=\frac{Q_{c}}{Q_{c}^{\max }}=\frac{v_{c}}{v_{c}^{\max }}
$$

where $Q_{c}^{\max }$ is the maximum capacity of the capacitor corresponding to the maximum voltage, $v_{c}^{\max }$, that can be tolerated by the capacitor. In order to simplify the description of the supervisory control system design in Section III, we also introduce the concept of depth of discharge of the battery bank and denote it as $d_{b}$, which is calculated as follows:

$$
d_{b}=1-s_{b} .
$$

The DC bus collects the energy generated by both the wind and solar subsystems and delivers it to the water desalination 
system and, if necessary, to the battery bank as well as to the electrical grid. The voltage of the DC bus is determined by the battery bank which comprises of lead-acid batteries.

Assuming an ideal voltage inverter, we can write an energy balance equation as follows:

$$
P_{w}+P_{s}=P_{R O}+i_{b} v_{b} .
$$

where $P_{R O}$ denotes the total power demand from the water desalination system. Note that when $i_{b}$ is positive, the battery bank is being charged and when $i_{b}$ is negative, the battery bank supplies energy to the RO water desalination system. The energy balance of Eq. 11 can be also expressed in the form of current balance as follows:

$$
i_{w}+i_{s}=i_{R O}+i_{b} .
$$

where $i_{R O}$ is the current injected to the RO water desalination system. From Eq. 12, we obtain the current across the battery bank as follows:

$$
i_{b}=i_{w}+i_{s}-i_{R O}
$$

The charge/discharge current of the battery bank $i_{b}$ will be used as a decision variable and optimally determined by the supervisory control system.

\section{B. Water desalination system description}

In the RO water desalination system, there is a highpressure pump, a membrane module and a water storage tank. Salt water enters the pump, which is equipped with a variable frequency drive, and is pressurized to the feed pressure [5]. The pressurized salt water stream enters the membrane module where it is separated into a low-salinity product (or permeate) stream, and a high-salinity brine (or retentate) stream. The permeate stream enters the storage tank which provides desalinated water to satisfy the water consumption demand.

In the RO system, the pressure downstream of the actuated valve and at the permeate outlet is assumed to be equal to atmospheric pressure. The RO system model is based on a mass balance taken around the entire system and an energy balance taken around the actuated retentate valve [5]:

$$
\frac{d v_{r}}{d t}=\frac{P_{s y s} A_{p}}{\rho_{w} V}-\frac{1}{2} \frac{A_{p} e_{v r} v_{r}^{2}}{V}
$$

where $v_{r}$ is the retentate stream velocity, $P_{\text {sys }}$ is the feed pressure, $A_{p}$ is the pipe cross-sectional area, $V$ is the system volume, $\rho_{w}$ is the fluid density, and $e_{v r}$ is the retentate valve resistance. The system pressure $P_{\text {sys }}$ can be calculated as follows:

$$
P_{s y s}=\frac{\rho_{w} A_{p}}{A_{m} K_{m}}\left(v_{f}-v_{r}\right)+\Delta \pi .
$$

where $A_{m}$ is the membrane area, $K_{m}$ is the membrane overall mass transfer coefficient, $v_{f}$ is the feed stream velocity, and $\Delta \pi$ is the difference in osmotic pressure between the feed side of the membrane and the permeate side and can be computed by [5]:

$$
\Delta \pi=\delta C_{\text {eff }}\left(T_{w}+273\right)
$$

where $C_{\text {eff }}$ is the effective average concentration at the membrane surface, $\delta$ is a constant relating effective concentration to osmotic pressure and $T_{w}$ is the water temperature in degrees Celsius.
Note that the three velocities $v_{f}, v_{r}$ and $v_{p}$ (the permeate stream velocity) satisfy an overall steady-state mass balance as follows (since all pipe cross-sectional areas are assumed to be the same):

$$
0=v_{f}-v_{r}-v_{p}
$$

Using the above dynamic equation, various control techniques can be applied using the valve resistance value $\left(e_{v r}\right)$ as the manipulated input.

In this work, we operate the RO system at energy optimal water recovery, $Y_{o p t}$, which implies that $\frac{v_{p}}{v_{f}}$ is being adjusted in real time; please see [1], [11] for discussions on how to compute and achieve the energy optimal water recovery in real time. Based on the Bernoulli equation and ignoring the water elevation change, we can obtain the power needed for the water desalination system as follows:

$$
P_{R O}=\frac{1}{\eta}\left(P_{s y s} \frac{F_{p}}{Y_{o p t}}+\frac{1}{2} \frac{F_{p}^{3}}{Y_{o p t}^{3} A_{p}^{2}} \rho_{w}\right), 0<\eta<1
$$

where $\eta$ is the overall power efficiency of the pump of the RO desalination system and $F_{p}=A_{p} v_{p}$ is the permeate flow rate (i.e., desalinated water production rate) which is used to satisfy the water consumption and storage demands. If we denote the water consumption demand as $F_{d}$ and water storage demand as $F_{s}$, then we obtain the following equation from a steady-state mass balance:

$$
0=F_{p}-F_{d}-F_{s} .
$$

Note that the water storage demand $F_{s}$ can take positive or negative values.

Based on the Eqs. 16 and 18, the dynamics of the water level in the storage tank, $h_{l}$, can be obtained as follows:

$$
\dot{h_{l}}=\frac{F_{s}}{A_{s}}=\frac{A_{p}}{A_{s}}\left(v_{f}-v_{r}\right)-\frac{F_{d}}{A_{s}}
$$

where $A_{s}$ is the cross-sectional area of the water storage tank. Similarly, we define the state of storage (SOS), $s_{t}$, for the storage tank as follows:

$$
s_{t}=\frac{h_{l}}{h_{l}^{\max }}
$$

where $h_{l}^{\max }$ is the maximum water level in the storage tank.

The dynamics of the integrated wind/solar/RO system can be written in the following compact form:

$$
\begin{aligned}
\dot{x} & =f(x)+g(x) u \\
h(x) & =0
\end{aligned}
$$

where $x=\left[\begin{array}{llllllll}i_{q} & i_{d} & \omega_{e} & v_{p v} & i_{s} & v_{c} & v_{r} & h_{l}\end{array}\right]^{T}, u=\left[\begin{array}{lll}u_{w} & u_{p v} & e_{v r}\end{array}\right]^{T}$, and $f, g, h$ are nonlinear vector functions whose explicit forms are omitted for brevity. We note that the dynamics of the integrated system exhibits a two-time-scale behavior. Specifically, the dynamics of the states, $i_{q}, i_{d}, w_{e}, v_{p v}, i_{s}$ and $v_{r}$, are relatively fast (in the order of seconds); and the dynamics of the states, $v_{c}$ and $h_{l}$, are relatively slow (in the order of minutes). Based on this two-time scale property, we can re-write the integrated system of Eq. 20 as follows:

$$
\begin{aligned}
\dot{x}_{f} & =f_{f}\left(x_{f}, x_{s}\right)+g_{f}\left(x_{f}, x_{s}\right) u \\
\dot{x}_{s} & =f_{s}\left(x_{f}, x_{s}\right) \\
h_{f}\left(x_{f}, x_{s}\right) & =0 \\
h_{s}\left(x_{f}, x_{s}\right) & =0
\end{aligned}
$$


where

$$
\begin{aligned}
f_{s}\left(x_{f}, x_{s}\right)= & {\left[\begin{array}{c}
\frac{1}{C_{b}} i_{b} \\
\frac{A_{p}}{A_{s}}\left(v_{f}-v_{r}\right)-\frac{F_{d}}{A_{s}}
\end{array}\right], } \\
h_{s}\left(x_{f}, x_{s}\right)= & {\left[\begin{array}{c}
P_{s y s}-\frac{\rho_{w} A_{p}}{A_{m} K_{m}}\left(v_{f}-v_{r}\right)-\Delta \pi \\
v_{f}-v_{r}-v_{p}
\end{array}\right] }
\end{aligned}
$$

and $x_{f}=\left[\begin{array}{llllll}i_{q} & i_{d} & w_{e} & v_{p v} & i_{s} & v_{r}\end{array}\right]^{T}, x_{s}=\left[\begin{array}{ll}v_{c} & h_{l}\end{array}\right]^{T}$. This two-time-scale property will be taken advantage of in the formulation of the supervisory MPC where the model of Eq. 21 with $\dot{x}_{f} \equiv 0$ will be used.

\section{CONTROL PROBLEM FORMULATION AND CONTROLLER DESIGN}

In this section, we design the supervisory control system to regulate the integrated energy generation and $\mathrm{RO}$ water desalination system. The primary control objective is to regulate the integrated system to produce enough desalinated water to satisfy the total water consumption and storage demands. The secondary objective is to take into account optimality considerations on system operation, for example, battery maintenance and energy savings. We propose to design the supervisory control system via MPC. Specifically, the MPC determines the operating power references $\left(P_{w}^{r e f}\right.$, $P_{s}^{r e f}$ ) for the wind and solar subsystems, the reference retentate flow rate $\left(v_{r}^{r e f}\right)$ for the RO water desalination subsystem, and the charge/discharge current $\left(i_{b}^{r e f}\right)$ of the battery bank. These trajectories are sent to the local controllers associated with the subsystems which force the subsystems to track the operating trajectories. For the designs of the local controllers, please refer to [9], [10], [5], [6].

In the MPC design, we explicitly account for considerations on the battery bank maintenance according to [12]. Specifically, we take into account the following considerations: 1) small charge/discharge currents are favorable, as large charge/discharge currents result in more energy dissipated in the battery internal resistance; 2) the charge current should be constrained under a certain upper bound which is a monotonically increasing function of the depth of discharge (DOD) of the battery bank. We set the upper bound of the charge currents based on a simple taper charging approach [12]; 3) The DOD of the battery bank should not exceed $d_{b}^{\max }$ in order to protect the battery bank, and 4) the battery should be charged if extra generated power is available because long periods in deep DOD are harmful to the battery.

To take into account energy savings considerations, we try to operate the $\mathrm{RO}$ water desalination subsystem at the energy optimal water recovery $Y_{\text {opt }}$ so that the energy consumption of unit water production is minimized [1]. In addition, we assume that there is a preferred SOS, $s_{t}^{o p t}$, of the storage tank which is a balance between the capacities of the tank to supply unexpected water consumption demand and to store extra water production. We consider the case where the future water consumption demand of the RO subsystem is known; that is, $F_{d}(t)$, is known. We also assume that future hourly weather conditions (i.e., wind speed, insolation, photovoltaic cell temperature) forecast information is available. These information will be used in the MPC to optimize the integrated system operation.

The supervisory MPC is evaluated at discrete time instants $t_{k}=t_{0}+k \Delta, k=0,1, \ldots$, with $t_{0}$ being the initial time and $\Delta$ being the sampling time. At each sampling time, piece-wise constant trajectories of the operating trajectories of the different subsystems (i.e., $P_{w}^{r e f}, P_{s}^{r e f}, v_{r}^{r e f}$ and $i_{b}^{r e f}$ ) for a certain time period (prediction horizon) are obtained but only the first piece of the trajectories are sent to the local controllers and implemented. Before we discuss the formulation of the supervisory MPC, we present the cost function used in the MPC. Specifically, the proposed form of the cost function is as follows:

$$
\begin{aligned}
J(t)= & \alpha \int_{t}^{t+N \Delta}\left(P_{R O}(\tau)+i_{b}^{r e f}(\tau) v_{b}(\tau)\right. \\
& \left.-P_{w}^{r e f}(\tau)-P_{s}^{r e f}(\tau)\right)^{2} d \tau \\
& +\beta \int_{t}^{t+N \Delta} \frac{P_{s}^{r e f}(\tau)}{P_{w}^{r e f}(\tau)} d \tau+\gamma \int_{t}^{t+N \Delta} d_{b}(\tau) d \tau \\
& +\epsilon \int_{t}^{t+N \Delta}\left(s_{t}(\tau)-s_{t}^{o p t}\right)^{2} d \tau+\zeta \int_{t}^{t+N \Delta} i_{b}^{r e f}(\tau)^{2} d \tau \\
& +\theta \frac{\int_{t}^{t+N \Delta} P_{R O}(\tau) d \tau}{\int_{0}^{N \Delta} F_{p}(\tau) d \tau}
\end{aligned}
$$

where $N$ is the prediction horizon of the MPC, $\alpha, \beta, \gamma, \epsilon, \zeta$ and $\theta$ are positive weighting factors. In this cost function, the first term is used to penalize the difference between the energy generated by the wind-solar system and the total power demand from the RO subsystem and the battery bank, thus driving the wind and solar subsystems to satisfy the total power demand to the maximum extent; the second term implies that the wind subsystem is operated as the primary generation subsystem and the solar subsystem is activated when necessary; the third term implies that the battery should be charged if the battery is not fully charged; the fourth term is used to make sure that the water level in the storage tank is maintained around the optimal water level; the fifth term takes into account that small charge currents are preferred; the sixth term penalizes the power consumption per unit of permeate water produced.

The proposed MPC design for the supervisory control system at time $t_{k}$ is as follows:

$$
\begin{array}{ll} 
& \min _{w}^{r e f}, P_{s}^{r e f}, i_{b}^{r e f}, v_{r}^{r e f} \in S(\Delta) \\
\text { s.t. } & 0 \leq P_{w}^{r e f}(\tau) \leq \min _{\tau}\left\{P_{w}^{\max }(\tau)\right\} \\
& 0 \leq P_{s}^{r e f}(\tau) \leq \min _{\tau}\left\{P_{s}^{\max }(\tau)\right\} \\
& F_{p}^{\min } \leq F_{p}(\tau) \leq F_{p}^{\max } \\
& 0 \leq d_{b}(\tau) \leq d_{b}^{\max } \\
& s_{t}^{\min } \leq s_{t}(\tau) \leq s_{t}^{\max } \\
& i_{b}^{r e f}(\tau) \leq i_{b}^{\max }\left(s_{b}(\tau)\right)
\end{array}
$$




$$
\begin{aligned}
& \dot{\tilde{x}}_{s}(\tau)=f_{s}\left(\tilde{x}_{f}(\tau), \tilde{x}_{s}(\tau)\right) \\
& 0=f_{f}\left(\tilde{x}_{f}(\tau), \tilde{x}_{s}(\tau)\right)+g_{f}\left(\tilde{x}_{f}(\tau), \tilde{x}_{s}(\tau)\right) u^{r e f} \\
& h_{s}\left(\tilde{x}_{f}(\tau), \tilde{x}_{s}(\tau)\right)=0 \\
& h_{f}\left(\tilde{x}_{f}(\tau), \tilde{x}_{s}(\tau)\right)=0 \\
& \tilde{x}_{s}(0)=x_{s}\left(t_{k}\right)
\end{aligned}
$$

where $S(\Delta)$ denotes the family of piece-wise constant functions, $\tau \in\left[t_{k+j}, t_{k+j+1}\right)$ with $j=0, \ldots, N-1, P_{w}^{\max }$ and $P_{s}^{\max }$ denote the maximum powers that can be achieved by the wind and solar subsystems, respectively, $\tilde{x}$ is the predicted future state trajectory of the integrated system, $x\left(t_{k}\right)$ is the state measurement obtained at time $t_{k}$, and $u^{r e f}$ denotes the steady-state control inputs obtained based on the optimal references computed by the MPC.

The constraints of Eqs. $23 \mathrm{~b}$ and $23 \mathrm{c}$ require that the computed wind and solar subsystems' power references should be smaller than the minimum of the maximum available within each sampling interval, which means that the power references should be achievable for the wind and solar subsystems. Note that the future maximum available power for the wind and the solar subsystems are estimated using the information of future weather conditions forecast. The constraint of Eq. 23d puts upper and lower bounds $\left(F_{p}^{\max }\right.$ and $F_{p}^{\min }$, respectively) on the permeate flow rate $F_{p}$, which is used to guarantee the equipment safety of the membrane module in the RO water desalination subsystem. The constraint of Eq. 23e requires that the depth of discharge of the battery bank should not exceed $d_{b}^{\max }$. The constraint of Eq. 23f imposes upper and lower bounds on the water level in the storage tank. The constraint of Eq. 23g places an upper bound on the charge current of the battery bank and this upper bound is a function of the current depth of discharge of the battery bank. Note that in the supervisory MPC design, only the slow system dynamics is taken into account (i.e., constraints of Eqs. 23h-231); and the fast system states that are (explicitly or implicitly) used in the MPC are estimated by the computed future operating trajectories (decision variables of the MPC). For example, in the calculation of future $F_{p}(\tau)$, the fast state $v_{r}$ is assumed to be equal to $v_{r}^{r e f}$ and then Eqs. 15 and 16 are solved; and in the calculation of future $d_{b}(\tau), i_{b}$ is assumed to be equal to $i_{b}^{r e f}$ in the evaluation of the system slow dynamics.

We denote the optimal solution to the optimization problem of Eq. 23 as $P_{w}^{r e f, *}\left(t \mid t_{k}\right), P_{s}^{r e f, *}\left(t \mid t_{k}\right), i_{b}^{r e f, *}\left(t \mid t_{k}\right)$ and $v_{r}^{r e f, *}\left(t \mid t_{k}\right)$. The references of power generation from the wind and solar subsystems, of battery charge/discharge current, and of RO retentate flow rate sent to the local controllers by the supervisory controller of Eq. 23 are defined as follows:

$$
\begin{aligned}
P_{w}^{r e f}(t) & =P_{w}^{r e f, *}\left(t \mid t_{k}\right), \forall t \in\left[t_{k}, t_{k+1}\right), \\
P_{s}^{r e f}(t) & =P_{s}^{r e f, *}\left(t \mid t_{k}\right), \forall t \in\left[t_{k}, t_{k+1}\right), \\
i_{b}^{r e f}(t) & =i_{b}^{r e f, *}\left(t \mid t_{k}\right), \forall t \in\left[t_{k}, t_{k+1}\right), \\
v_{r}^{r e f}(t) & =v_{r}^{r e f, *}\left(t \mid t_{k}\right), \forall t \in\left[t_{k}, t_{k+1}\right) .
\end{aligned}
$$

Note that the constraints of Eqs. 23b-231 are inspired by results on the design of Lyapunov-based model predictive control systems (please see [13], [14]).

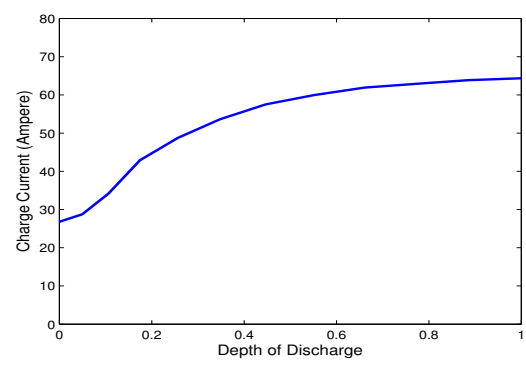

Fig. 2. Upper bound on battery charge current.

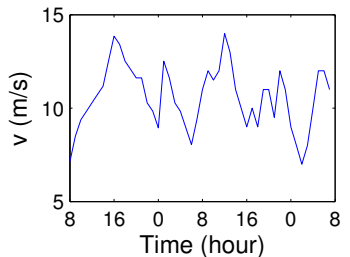

(a)

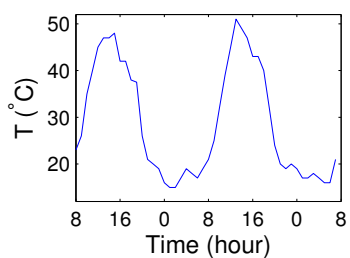

(c)

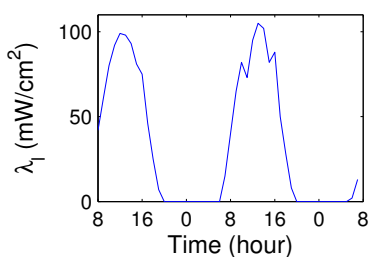

(b)

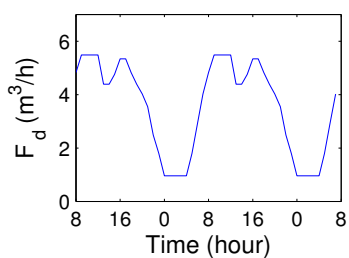

(d)
Fig. 3. Forecast of weather conditions and water demand. (a) Wind speed $v$; (b) insolation $\lambda_{l}$; (c) PV panel temperature $T$ and (d) water demand $F_{d}$.

\section{Simulation Results}

The prediction horizon and the sampling time of the MPC are chosen to be $N=24$ and $\Delta=1 \mathrm{hr}$ taking into account that the water demand (for example, of a community) usually presents periodic fluctuations with a period of 24 hours. The weighting factors in the cost function are chosen to be $\alpha=1.8 \times 10^{-7}, \beta=0.001, \gamma=0.001, \epsilon=0.01$, $\zeta=0.05 \times 10^{-9}$ and $\theta=2 \times 10^{-7}$. We carefully size the capacity of the units to match each other and to moderately exceed the average water demand. The overall RO system pump power efficiency is assumed to be $\eta=0.7$, the upper bound on $d_{b}$ is $d_{b}^{\max }=0.8$, the lower and upper bounds on $F_{p}$ are $F_{p}^{\min }=0.1814 \mathrm{~m}^{3} / \mathrm{hr}$ and $F_{p}^{\max }=3.9918 \mathrm{~m}^{3} / \mathrm{hr}$, respectively, and the lower and upper bounds on $s_{t}$ are $s_{t}^{\min }=0$ and $s_{t}^{\max }=1$, respectively. The upper bound on the battery charge current is a function of $d_{b}$ and is shown in Fig. 2.

We carry out simulations for one day starting at $8 \mathrm{am}$. In the simulations, we assume that weather and water demand forecast information of future 24 hours is available. A twoday forecast of wind speed, insolation, PV cell temperature and water demand is shown in Fig. 3, which is used by MPC to estimate the future 24-hour environmental variation at each sampling time. We introduce hourly deviation and high frequency disturbances to the forecast information to simulate realistic fluctuations of weather and water demand for the first 24 hours, as shown in Fig. 4.

Fig. 5 shows the time evolution of wind and solar sub- 


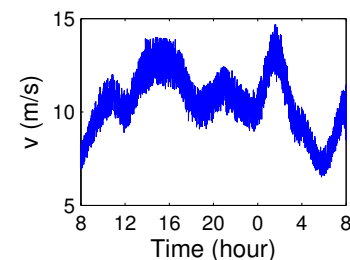

(a)

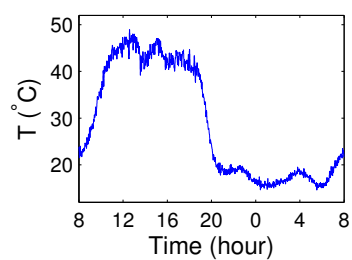

(c)

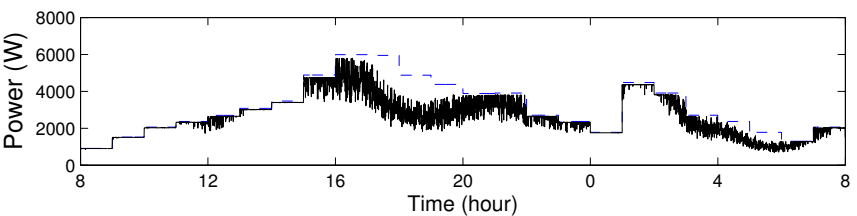

(a)

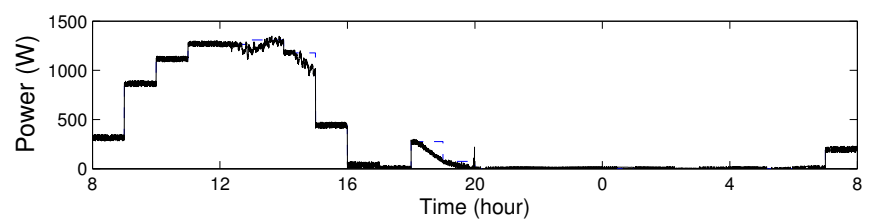

(b)

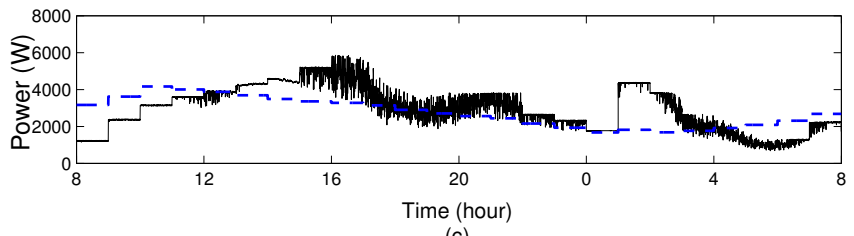

Fig. 5. Power generation and consumption in the stand-alone operating mode. (a) Power generated by wind subsystem $P_{w}$ (solid line) and wind power reference $P_{w, \text { ref }}$ (dashed line). (b) Power generated by solar subsystem $P_{s}$ (solid line) and solar power reference $P_{s, \text { ref }}$ (dashed line). (c) Generated power $P_{w}+P_{s}$ (solid line) and total power demand $P_{R O}$ (dashed line).

system power generation and RO subsystem power consumption. For each hour of operation, the wind/solar local controllers operate to drive the wind mill and the PV panel array to generate power according to the reference values, respectively. However, when the weather condition does not permit sufficient generation, for example during $15 \sim 21 \mathrm{hr}$ and during $3 \sim 7 \mathrm{hr}$ for the wind subsystem as shown in Fig. 5(a), the local controller switches to the operation mode of maximum power point tracking. In Fig. 5(c), when wind/solar power delivery alone cannot meet the RO power demand such as during $8 \sim 13 h r$ and during $4 \sim 8 h r$, the battery discharges to compensate for the shortfall; at other times, extra power generation is used to charge the battery.

An advantage of the supervisory MPC is that it is able to schedule the water production to be smooth (nearly uniform with respect to time) by taking into account future state variation and by coordinating the subsystems. It can be seen from Fig. 6(a) that water production is relatively smooth

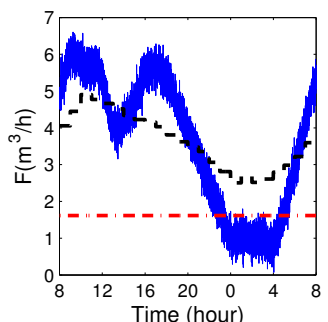

(a)

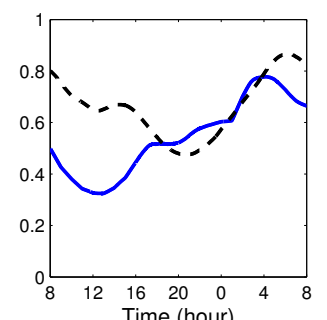

(b)
Fig. 6. (a) Rate of water demand $F_{d}$ (solid line), permeate flow rate $F_{p}$ (dashed line) and the flow rate corresponding to the minimum power consumption per unit water production (dashed-dotted line). (b) Battery state of charge $s_{b}$ (solid line) and tank state of storage $s_{t}$ (dashed line).

despite dramatically varying water demand and weather conditions during a day. This is largely due to the optimized utilization of the capacities of the battery bank and of the water tank which act as buffers against external fluctuations. The state of charge of the battery bank and the state of storage of the water tank are shown in Fig. 6(b).

\section{REFERENCES}

[1] A. Zhu, A. Rahardianto, P. D. Christofides, and Y. Cohen, "Reverse osmosis desalination with high permeability membranes - cost optimization and research needs," Desalination and Water Treatment, vol. 15, pp. 256-266, 2010.

[2] C. Charcosset, "A review of membrane processes and renewable energies for desalination," Desalination, vol. 245, pp. 214-231, 2007.

[3] K. Tan and S. Islam, "Optimum control strategies in energy conversion of PMSG wind turbine system without mechanical sensors," IEEE Transactions on Energy Conversion, vol. 19, pp. 392-399, 2004.

[4] N. Hamrouni, M. Jraidi, and A. Cherif, "New control strategy for 2stage grid-connected photovoltaic power system," Renewable Energy, vol. 33, pp. 2212-2221, 2008.

[5] A. R. Bartman, P. D. Christofides, and Y. Cohen, "Nonlinear modelbased control of an experimental reverse-osmosis water desalination system," Industrial and Engineering Chemistry Research, vol. 48, pp. 6126-6136, 2009.

[6] W. Qi, J. Liu, X. Chen, and P. D. Christofides, "Supervisor predictive control of stand-alone wind-solar energy generation systems," IEEE Transactions on Control Systems Technology, vol. 19, pp. 199-207, 2011.

[7] W. Qi, J. Liu, X. Chen, and P. D. Christofides, "Supervisory Predictive Control of an Integrated Wind/Solar Energy Generation and Water Desalination System," in Proceedings of the 9th International Symposium on Dynamics and Control of Process Systems, Leuven, Belgium, 2010, pp. 821-826.

[8] D. Q. Mayne, J. B. Rawlings, C. V. Rao, and P. O. M. Scokaert, "Constrained model predictive control: Stability and optimality," $\mathrm{Au}$ tomatica, vol. 36, pp. 789-814, 2000.

[9] F. Valenciaga, P. F. Puleston, P. E. Battaiotto, and R. J. Mantz, "Passivity/sliding mode control of a stand-alone hybrid generation system," IEE Proceedings on Control Theory and Applications, vol. 147, pp. 680-686, 2000.

[10] F. Valenciaga, P. F. Puleston, and P. E. Battaiotto, "Power control of a photovoltaic array in a hybrid electric generation system using sliding mode techniques," IEE Proceedings on Control Theory and Applications, vol. 148, pp. 448-455, 2001.

[11] A. Zhu, P. D. Christofides, and Y. Cohen, "Effect of thermodynamic restriction on energy cost optimization of RO membrane water desalination," Industrial and Engineering Chemistry Research, vol. 48, pp. 6010-6021, 2009.

[12] D. Linden and T. B. Reddy, Eds., Handbook of batteries, 3rd ed. McGraw-Hill, 2002.

[13] P. D. Christofides and N. H. El-Farra, Control of nonlinear and hybrid process systems: Designs for uncertainty, constraints and time-delays. Berlin, Germany: Springer-Verlag, 2005.

[14] P. Mhaskar, N. H. El-Farra, and P. D. Christofides, "Stabilization of nonlinear systems with state and control constraints using Lyapunovbased predictive control," Systems and Control Letters, vol. 55, pp. 650-659, 2006. 